\title{
Características físicas e químicas dos solos do Perímetro Irrigado Cachoeira II em função do tempo
}

\author{
Physical and chemical characteristics of soils of the Irrigated \\ Perimeter Cachoeira II in function of time
}

Josimar Gurgel Fernandes ${ }^{*}$; Maria Betânia Galvão dos Santos Freire ${ }^{2}$, Josiclêda Domiciano Galvincio $^{3}$. Patrícia Ribeiro dos Santos ${ }^{4}$, Jailson Cavalcante Cunha ${ }^{5}$

${ }^{1}$ Instituto Agronômico de Pernambuco (IPA), Av. Gen. San Martin, 1371. Bongi, CEP 50761-000, Recife, PE, Brasil

${ }^{2}$ Departamento de Agronomia, Universidade Federal Rural de Pernambuco (UFRPE), Recife, PE, Brasil Departamento de Geografia Universidade Federal de Pernambuco (UFPE), Recife, PE, Brasil ${ }^{4}$ Instituto Federal de Pernambuco (IFPE), Barreiros, PE, Brasil

${ }^{5}$ Programa de Pós-Graduação em Solos e Nutrição de Plantas, Universidade Federal de Viçosa (UFV), Viçosa, PE Brasil

* autor correspondente 凶 josimar.gurgel@ipa.br
RESUMO: O objetivo deste trabalho foi verificar as alterações nas características físico-químicas dos solos do Perímetro Irrigado Cachoeira II que contribuem para a salinização e sodificação dos solos e sua variação ao longo do ano. As coletas de solos foram realizadas trimestralmente nas profundidades de 0-20, 20-40 e 40-60 cm, iniciando em junho de 2006 e finalizando em março de 2007. Nestas amostras, foram realizadas leituras de $\mathrm{pH}$ em água; determinações dos sais solúveis no extrato de saturação - $\mathrm{CE}$, cátions $\left(\mathrm{Ca}^{2+}\right.$, $\mathrm{Mg}^{2+}, \mathrm{Na}^{+}$e $\left.\mathrm{K}^{+}\right)$e ânions $\left(\mathrm{Cl}^{-}, \mathrm{HCO}_{3}-\mathrm{e} \mathrm{CO}_{3}{ }^{2-}\right)$. Foram também determinados os cátions trocáveis $\left(\mathrm{Ca}^{2+}, \mathrm{Mg}^{2+}, \mathrm{Na}^{+}\right.$e $\left.\mathrm{K}^{+}\right)$e calculadas a RAS (relação de adsorção de sódio) e a PST (percentagem de saturação de sódio) dos solos. A caracterização física foi realizada na amostragem de solo da primeira coleta. Com a análise dos dados, foi possível observar que os solos apresentam uma boa fertilidade natural e os valores de $\mathrm{pH}$ variaram ao longo do ano em estudo, ficando na faixa entre neutralidade e alcalinidade. Foi observado um incremento no teor de $\mathrm{Na}^{+}$trocável e da PST com a profundidade nos solos do perímetro, sendo esse aumento da PST suficiente para causar limitações no uso do solo. Para os cátions e ânions solúveis estudados, as maiores concentrações foram registradas nas camadas superiores, possivelmente pela ascensão capilar da água com sais e elevada evaporação em superfície. Verificou-se, ainda, que aproximadamente $12 \%, 14 \%$ e $28 \%$ da área estudada estão afetadas por sais, nas profundidades de 0-20, 20-40 e 40-60 cm, respectivamente.

PALAVRAS-CHAVE: Solos afetados por sais, PST, semiárido.
ABSTRACT: The aim of this study was to verify changes in the physicochemical properties of the soil under the 'Cachoeira II' Irrigated Perimeter, which contribute to salinization and sodification, and their variation over time. Soil samples were collected every three months at depths of 0-20, 20-40 and 40-60 cm from June 2006 to March 2007. In these samples, we performed readings of $\mathrm{pH}$ in water and determination of soluble salts in saturation extract - SE: cations $\left(\mathrm{Ca}^{2+}, \mathrm{Mg}^{2+}\right.$, $\mathrm{Na}^{+}$and $\left.\mathrm{K}^{+}\right)$and anions $\left(\mathrm{Cl}^{-}, \mathrm{HCO}_{3}\right.$ - and $\mathrm{CO}_{3}{ }^{2-}$ ). We also extracted the exchangeable cations $\left(\mathrm{Ca}^{2+}, \mathrm{Mg}^{2+}, \mathrm{Na}^{+}\right.$and $\left.\mathrm{K}^{+}\right)$and calculated the sodium adsorption ratio (SAR) and exchangeable sodium percentage (ESP) of the soil. Physical characterization was performed in the soil of the first collection. Data analysis showed that the soil presented good natural fertility and varying values of $\mathrm{pH}$ throughout the study period, ranging between neutrality and alkalinity. Exchangeable $\mathrm{Na}^{+}$concentration and ESP increased with depth in the irrigated perimeter soils, and this increase in PST was sufficient to limit land use. Regarding the soluble cations and anions studied, the highest concentrations were verified in the upper layers, possibly owing to capillary rise of water with salts and high evaporation. We also observed that approximately 12, 14 and $28 \%$ of the study area was affected by salt at depths of 0-20, 20-40 and 40-60 cm, respectively.

KEYWORDS: Salt-affected soils, PST, semiarid region. 


\section{Introdução}

Solos de regiões áridas e semiáridas podem apresentar acúmulo de sais prejudicial ao crescimento das plantas, decorrente de processos naturais de salinização ou da contribuição humana, pelo manejo inadequado da irrigação. Os sais em excesso prejudicam o crescimento das plantas não só pelos efeitos diretos sobre o potencial osmótico da solução do solo e pelos íons potencialmente tóxicos presentes em elevadas concentrações, mas também pela degradação de algumas propriedades físicas, reduzindo a infiltração da água, trocas gasosas, crescimento de raízes e, com isso, dificultando o crescimento das plantas. A salinização dos solos é um dos grandes problemas em muitas outras regiões do mundo, em razão, principalmente, da intensa evapotranspiração, baixas precipitações e irrigação (SILVEIRA et al., 2001).

São muitos os exemplos de salinização em perímetros irrigados, em virtude de irrigação inadequada. Conforme Batista et al. (2001), a salinização decorrente da irrigação tem sido observada em regiões onde a precipitação pluviométrica anual é inferior a $1.000 \mathrm{~mm}$, em particular em solos rasos e mal drenados.

Em sua grande maioria os perímetros irrigados são instalados em solos de boa fertilidade natural, localizados próximos aos mananciais de água, com facilidade de acesso à irrigação e, portanto com boa aptidão agrícola, sendo bastante representativos para a economia da região. Entretanto, podem tornar-se subutilizados se não forem bem manejados. Isso se deve à elevação da concentração salina na solução do solo e a problemas físicos dos solos, que podem excluir essas áreas do processo produtivo.

Estudos realizados por Sousa (1995) em solos aluviais do Vale do Pajeú, em Serra Talhada (PE), encontraram predomínio de plagioclásio sódico na mineralogia das frações areia e silte, mais especificamente na areia fina, indicando ser um problema regional que pode propiciar a sodificação pela liberação do sódio ao sistema. De acordo com o autor, embora os teores apresentem-se relativamente baixos, principalmente na camada superficial dos solos, existe uma tendência de acúmulo de sais resultante da presença de plagioclásio no material de origem, indicando assim que os solos da região apresentam uma tendência natural ao processo de salinização se não forem bem manejados.

Segundo Freire e Freire (2007), as áreas salinas e sódicas só deixarão de se expandir no Brasil quando houver maior preocupação com os efeitos da irrigação sobre a capacidade produtiva dos solos, com a adoção de práticas de manejo adequadas para evitar a salinização de locais ainda não afetados pelo problema, e também com a melhoria das condições dos solos já afetados.

Apesar dos avanços tecnológicos, milhões de hectares continuam sendo salinizados contribuindo severamente para a redução da produção agrícola mundial (KHAN; ABDULLAH, 2003). Estima-se que aproximadamente um terço da área irrigada mundial se apresenta degradada em virtude da salinidade e sodicidade de solos (GHASSEMI; JAKEMAN; NIX, 1995). Contudo, não existem muitos dados de áreas degradadas pela salinização e sodificação de solos em perímetros irrigados do Nordeste do Brasil. Esta quantificação possibilitaria vislumbrar a extensão de áreas afetadas por sais, quais as principais causas do problema, para tentar conter seu crescimento.

Este trabalho teve como objetivo verificar as alterações nas características físicas e químicas dos solos do Perímetro Irrigado Cachoeira II, que contribuem para a salinização e sodificação dos solos e sua variação ao longo de um ano.

\section{Material e Métodos}

Segundo a classificação de Köppen, adaptada no Brasil, a área está situada no domínio do clima Bsw'h', muito quente e semiárido que compreende o Perímetro Irrigado Cachoeira II, composto por 37 lotes, grande parte em solos aluvial (Neossolos Fluvicos), localizado no município de Serra Talhada, no semiárido de Pernambuco, mesorregião do Sertão do Alto Pajeú. O perímetro situa-se nas coordenadas geográficas $7^{\circ} 58^{\prime} 54$ " a $8^{\circ} 01$ ' 36" de Latitude Sul e $38^{\circ} 18^{\prime}$ $24^{\prime \prime}$ a $38^{\circ} 21^{\prime} 21^{\prime \prime}$ de Longitude Oeste, ficando a jusante do açude Cachoeira II (DEPARTAMENTO..., 1999).

Foram estudados os solos dos 37 lotes que compõem o Perímetro, tomando-se uma amostra por lote em ponto georreferenciado para que todas as amostragens coincidissem no mesmo local. Essas amostragens foram trimestrais nas profundidades de 0-20, 20-40 e 40-60 cm, sendo as amostras secas ao ar, destorroadas e passadas em peneira de $2 \mathrm{~mm}$ para a caracterização física e química dos solos. A primeira coleta de solo foi realizada no mês de junho de 2006, a segunda em setembro de 2006, a terceira foi realizada em dezembro de 2006 e a quarta e última coleta no mês de março de 2007.

Nestas amostras, foram realizadas leituras de $\mathrm{pH}$ em água na relação 1:2,5 solo:água, determinações dos sais solúveis no extrato de saturação - CE (condutividade elétrica), cátions $\left(\mathrm{Ca}^{2+}, \mathrm{Mg}^{2+}, \mathrm{Na}^{+}\right.$e $\left.\mathrm{K}^{+}\right)$e ânions $\left(\mathrm{Cl}^{-}, \mathrm{HCO}_{3}-\mathrm{e} \mathrm{CO}_{3}{ }^{2-}\right)$. Foram também determinados os cátions trocáveis $\left(\mathrm{Ca}^{2+}, \mathrm{Mg}^{2+}, \mathrm{Na}^{+}\right.$ e $\mathrm{K}^{+}$) extraídos com solução de acetato de amônio $1 \mathrm{~mol} \mathrm{~L}^{-1}$, sendo o potássio e o sódio dosados por fotometria de emissão de chama; e o cálcio e o magnésio dosados por espectrofotometria de absorção atômica (RICHARDS, 1954). A CTC (capacidade de troca de cátions) dos solos foi determinada pelo método do acetato de sódio/acetato de amônio, segundo recomendações de Richards (1954). Com os resultados dos elementos solúveis, foi calculada a RAS (relação de adsorção de sódio) e, com os elementos trocáveis, a PST (percentagem de sódio trocável) dos solos (RICHARDS, 1954).

A caracterização física foi realizada nos solos de uma das amostragens, constando de análise granulométrica pelo método da pipeta, condutividade hidráulica em meio saturado com o permeâmetro de coluna vertical e carga constante, umidade na capacidade de campo e ponto de murcha permanente pelo método do extrator de Richards (EMPRESA..., 1997). Também foi analisada a argila dispersa em água pelo método da pipeta; a densidade do solo pelo método do anel volumétrico; a densidade das partículas pelo método do balão volumétrico; todos seguindo métodos descritos pela EMBRAPA (EMPRESA..., 1997).

Para verificar a aderência ou não dos dados à distribuição normal, aplicou-se o teste de Komolgorov-Smirnov (KS) ao 
nível de $1 \%$ e $5 \%$ de probabilidade. Os resultados obtidos foram inicialmente analisados por meio de técnicas estatísticas descritivas, ou seja, por meio de cálculos da média, mediana e moda. Foram também calculados os valores máximo e mínimo dessas propriedades, o desvio padrão (DP), o coeficiente de variação $(\mathrm{CV})$ e os valores de curtose. De acordo com os valores de CV, a variabilidade dessas propriedades foi classificada, segundo Warrick e Nielsen (1980), em baixa $(C V<12 \%)$, média $(12<\mathrm{CV}<62 \%)$ e alta $(\mathrm{CV}>62 \%)$.

\section{Resultados e Discussão}

$\mathrm{O}$ valor da condutividade hidráulica no meio saturado $\left(\mathrm{K}_{0}\right)$ no perfil do solo foi variável, diferindo entre as camadas de solo (Quadro 1). Observa-se que a condutividade hidráulica saturada da camada do solo de $0-20 \mathrm{~cm}$, varia de 0,00 a $6,22 \mathrm{~cm}$ $\mathrm{h}^{-1}$, com média igual a $2,02 \mathrm{~cm} \mathrm{~h}^{-1}$, e desvio padrão de $1,83 \mathrm{~cm}$ $\mathrm{h}^{-1}$; nas camadas de 20-40 e 40-60 cm verificou-se tendência de aumento da $\mathrm{K}_{0}$ com a profundidade, principalmente na última camada avaliada.

Esses dados vêm corroborar os encontrados por Barreto et al. (2001), que constataram a existência de grandes variações espaciais, horizontal e vertical, nos valores da condutividade hidráulica saturada, em um solo aluvial do Perímetro Irrigado de São Gonçalo - PB.

A princípio, a variação constatada na condutividade hidráulica pode ser atribuída, em parte, à gênese e evolução do solo estudado, que aconteceram por meio de processos de sedimentação aluvial durante o seu desenvolvimento

Quadro 1. Medidas descritivas das análises físicas dos solos do Perímetro Irrigado Cachoeira II, Serra Talhada - PE em junho de 2006.

\begin{tabular}{|c|c|c|c|c|c|c|c|c|}
\hline \multirow{2}{*}{ Medidas } & $D s^{1}$ & $\mathrm{CC}^{2}$ & $\mathbf{P M P}^{3}$ & $\mathbf{K}_{0}{ }^{4}$ & $\mathbf{A G}^{5}$ & $\mathbf{A F}^{6}$ & Silte & Argila \\
\hline & $\mathrm{kg} \mathrm{dm}^{-3}$ & $\mathbf{m}^{3} \mathbf{m}^{3}$ & $\mathbf{m}^{3} \mathbf{m}^{3}$ & $\mathbf{c m ~ h}^{-1}$ & $\mathrm{~kg} \mathrm{~kg}^{-1}$ & $\mathrm{~kg} \mathrm{~kg}^{-1}$ & $\mathrm{~kg} \mathrm{~kg}^{-1}$ & $\mathrm{~kg} \mathrm{~kg}^{-1}$ \\
\hline \multicolumn{9}{|c|}{$0-20 \mathrm{~cm}$} \\
\hline Média & 0,12 & 0,14 & 0,05 & 2,02 & 0,0967 & 0,4244 & 0,4698 & 0,0091 \\
\hline Mediana & 0,12 & 0,13 & 0,05 & 1,66 & 0,0580 & 0,4550 & 0,4477 & 0,0075 \\
\hline Moda & $n e^{9}$ & ne & ne & 0,00 & 0,0265 & ne & ne & ne \\
\hline $\mathrm{DP}^{7}$ & 0,07 & 0,00 & 0,02 & 1,83 & 0,0901 & 0,1232 & 0,1212 & 0,0080 \\
\hline $\mathrm{CV}^{8}$ & 58,47 & 32,79 & 42,10 & 90,41 & 93,1000 & 29,0200 & 25,8000 & 88,6500 \\
\hline Curtose & 141,04 & 0,67 & 1,79 & $-0,13$ & 2,3000 & $-0,6000$ & 0,9900 & 32,7200 \\
\hline Máxima & 0,33 & 0,25 & 0,12 & 6,22 & 0,3850 & 0,6615 & 0,7980 & 0,0552 \\
\hline Mínima & 0,02 & 0,08 & 0,01 & 0,00 & 0,0130 & 0,1730 & 0,2713 & 0,0049 \\
\hline \multicolumn{9}{|c|}{$20-40 \mathrm{~cm}$} \\
\hline Média & 0,17 & 0,14 & 0,06 & 2,04 & 0,9312 & 4,8135 & 4,8135 & 0,0831 \\
\hline Mediana & 0,16 & 0,13 & 0,06 & 1,32 & 0,6150 & 4,2650 & 4,5690 & 0,0760 \\
\hline Moda & ne & ne & ne & 0,00 & 0,0265 & 5,4750 & ne & ne \\
\hline $\mathrm{DP}^{7}$ & 0,06 & 0,05 & 0,02 & 1,98 & 0,9029 & 1,4660 & 1,4656 & 0,0250 \\
\hline $\mathrm{CV}^{8}$ & 36,26 & 0,36 & 0,40 & 97,05 & 0,9696 & 0,3045 & 0,3045 & 0,3005 \\
\hline Curtose & 103,67 & 0,00 & 0,03 & $-0,22$ & 0,0147 & $-0,0072$ & 0,0052 & 0,0108 \\
\hline Máxima & 0,34 & 0,27 & 0,13 & 6,30 & 3,5700 & 6,6800 & 8,7154 & 0,1529 \\
\hline Mínima & 0,05 & 0,07 & 0,02 & 0,00 & 0,04 & 1,0350 & 1,8251 & 0,0450 \\
\hline \multicolumn{9}{|c|}{$40-60 \mathrm{~cm}$} \\
\hline Média & 0,21 & 0,15 & 0,06 & 2,40 & 0,8646 & 4,0043 & 5,0403 & 0,0908 \\
\hline Mediana & 0,19 & 0,14 & 0,06 & 0,89 & 0,5000 & 4,0500 & 4,6915 & 0,0898 \\
\hline Moda & ne & ne & ne & 0,00 & ne & ne & ne & ne \\
\hline $\mathrm{DP}^{7}$ & 0,06 & 0,06 & 0,03 & 4,17 & 0,0184 & 1,8428 & 1,8428 & 0,0322 \\
\hline $\mathrm{CV}^{8}$ & 30,48 & 0,39 & 0,45 & 173,63 & 1,0623 & 0,4616 & 0,3656 & 0,3535 \\
\hline Curtose & 56,08 & 0,00 & 0,02 & 11,14 & 0,0107 & $-0,0053$ & $-0,0037$ & 0,0051 \\
\hline Máxima & 0,37 & 0,29 & 0,14 & 20,96 & 3,5350 & 8,2300 & 8,8619 & 0,1710 \\
\hline Mínima & 0,08 & 0,04 & 0,02 & 0,00 & 0,005 & 0,8250 & 1,3565 & 0,0335 \\
\hline
\end{tabular}

${ }^{1} \mathrm{Ds}=$ Densidade do solo $;{ }^{2} \mathrm{CC}=$ Capacidade de Campo; ${ }^{3} \mathrm{PMP}$ Ponto de Murcha Permanente; ${ }^{4} \mathrm{~K}_{0}=$ Condutividade hidráulica em meio saturado; ${ }^{5} \mathrm{AG}=\mathrm{Areia}$ Grossa; ${ }^{6} \mathrm{AF}=$ Areia Fina $;{ }^{7} \mathrm{DP}=$ Desvio padrão $;{ }^{8} \mathrm{CV}=$ Coeficiente de variação $;{ }^{9}$ ne = valor não encontrado. 
hidrogeológico, ocasionando assim a deposição de camadas e composições físicas variáveis.

A redução da infiltração da água no solo promovida pelos sais de sódio dificulta a troca de gases entre o solo e o ar atmosférico, comprometendo a reposição de lâminas de água e afetando adversamente as culturas irrigadas. Em trabalho realizado com solos de Pernambuco, FREIRE et al. (2003a), constataram que, quando o sódio é o íon predominante, os solos se tornam impermeáveis e isso compromete seu uso na agricultura irrigada. Esta redução pode alcançar tal magnitude que as raízes das plantas podem não receber água em quantidade suficiente entre os turnos de rega.

Constatou-se que o teor médio de água no solo na camada de $0-20 \mathrm{~cm}$ é de $0,14 \mathrm{~m}^{3} \cdot \mathrm{m}^{3}$ e $0,05 \mathrm{~m}^{3} \cdot \mathrm{m}^{3}$ para a capacidade de campo (CC) e o ponto de murcha permanente (PMP), respectivamente, o que perfaz uma quantidade de $8,4 \%$ de água disponível. O coeficiente de variação (CV) foi de $32,79 \%$ para CC e $42,1 \%$ para PMP na camada de $0-20 \mathrm{~cm} ; 35,8 \%$ para CC e 40,49\% para PMP na camada de $20-40 \mathrm{~cm}$; e $39,28 \%$ para CC e $44,86 \%$ para PMP na camada de $40-60 \mathrm{~cm}$, sendo esses resultados classificados como CV médio, segundo critérios estabelecidos por Warrick e Nielsen (1980). O teor de água disponível nas camadas de $20-40$ e $40-60 \mathrm{~cm}$ foi de $8,62 \%$ e $8,77 \%$, respectivamente (Quadro 1).

A densidade do solo (Ds) apresentou variações consideráveis entre as amostragens de solo nos lotes, como já era de se esperar, devido à natureza de formação desses solos (solos aluvionais). Assim sendo, podemos observar que ocorre um acréscimo nos valores médios da Ds com relação à profundidade de amostragem, assim como a mediana. A amplitude de variação, valores de máximos e mínimos é considerável entre as amostragens nos lotes, isso faz com que o $\mathrm{CV}$ apresente valores elevados para essa variável analisada, segundo os critérios estabelecidos por Warrick e Nielsen (1980).

A composição granulométrica de todos os solos do Perímetro revelou a predominância de silte e areia, principalmente areia fina, com reduzidos teores de argila. Isso influencia sobremaneira na infiltração de água e ar no perfil do solo, pois a areia fina e o silte são partículas finas que podem promover a obstrução de poros, formando camadas mais adensadas em profundidade, dificultando a passagem de água e ar, contribuindo para os reduzidos valores de $\mathrm{K}_{0}$ observados (Quadro 1).

Existe um acréscimo de concentração da fração silte nas camadas mais profundas de amostragem em detrimento da concentração de areia (areia grossa e areia fina). A elevada concentração de areia e silte nesses solos deve-se, em parte, às sucessivas deposições de camadas de sedimentos trazidas pelo rio em épocas distintas, que podem variar em constituição (COELHO; FERREYRA, 1986).

Constatou-se grande amplitude de variação para o $\mathrm{pH}$ e a CE dos solos, com base nos valores máximos e mínimos (Quadro 2). No entanto, essa amplitude deve ser analisada com certas restrições, já que considera apenas os dois valores extremos de um conjunto de dados, sendo, muitas vezes, afetada por um valor particularmente discrepante, concordando com Salviano, Vieira e Sparovek (1998). Com a aplicação do teste de Kolmogorov-Smirnov, foi observado que as amostras foram normais e significativas a $1 \%$ ou a $5 \%$.

Os valores médios de $\mathrm{pH}$ variaram ao longo do ano em estudo, o mesmo ocorrendo para os valores máximos e mínimos nas amostras das três camadas avaliadas (Quadro 2). Analisando os valores de máximo e mínimo na camada de 0-20, observa-se que os solos do perímetro apresentam o $\mathrm{pH}$ na faixa de 6,50 a 9,60, ou seja, próximos à neutralidade, tendendo a alcalinos (EMATER, 1979). Resultados semelhantes foram encontrados em uma área do Perímetro Irrigado Senador Nilo Coelho, Petrolina/PE por Silva (2001). No entanto, os valores da média e mediana, nas amostras de $0-20 \mathrm{~cm}$ demonstraram que na área estudada predominam solos com $\mathrm{pH}$ em torno de 8,1, ou seja, alcalinos, concordando com Cavalcanti et al. (1999). Estes valores de $\mathrm{pH}$ em profundidade vão aumentando, caracterizando-se em um fator negativo para o desenvolvimento de plantas, considerando-se que a faixa de $\mathrm{pH}$ ideal vai de 6,0 a 6,5 para a maioria das culturas, concordando assim com os dados obtidos por Souza, Queiroz e Gheyi (2000).

Analisando a frequência dos resultados, verifica-se que é desnecessário o uso de corretivo para acidez, uma vez que os valores de $\mathrm{pH}$ são elevados, todavia, este $\mathrm{pH}$ elevado tende a dificultar ou impossibilitar a absorção de alguns elementos necessários ao bom desenvolvimento das culturas.

Com base na classificação de Warrick e Nielsen (1980), verifica-se baixa variação, com $\mathrm{CV}$ de $5,23 \%$ a $7,89 \%$ para o pH, mas elevada para a CEes, com CV entre $192,77 \%$ e 279,02\% na profundidade de 0-20 cm (Quadro 2). Pode-se também observar uma tendência de diminuição no coeficiente de variação para essas variáveis à medida que aumenta a profundidade do solo, o que infere um caráter heterogêneo ao solo da área, próprio de Neossolos Flúvicos (solos aluvionais), concordando com Oliveira (1998), Ceuppens e Wopereis (1999), Souza, Queiroz e Gheyi (2000), Gonçalves et al. (2011) e Andrade et al. (2012).

A distribuição dos teores de cálcio e de magnésio trocável dos solos variou dentro dos padrões normais para estes solos, como pode ser observado pelos valores da média, mediana e moda (Quadro 3). A maior parte das amostras de solo do presente trabalho apresentou teores de cálcio e de magnésio com uma amplitude de variação média a baixa, apresentando um CV classificado como médio entre as quatro coletas e as três profundidades de amostragem.

Com relação aos teores de potássio, pode-se verificar uma forte tendência à diminuição da concentração do teor de potássio nas camadas mais profundas do solo. Levando em consideração a concentração de potássio no solo, principalmente na camada agricultável, não se faz necessária à recomendação de adubação para este elemento, tendo em vista sua alta a muito alta concentração no solo, conforme critérios estabelecidos pela EMBRAPA (EMPRESA..., 1980).

A evolução vertical dos teores de sódio e da PST nos solos do perímetro permite inferir que há um aumento da sodicidade com a profundidade, assim sendo, observa-se que o aumento na PST chega a ser suficiente para causar limitações no uso do solo, visto que os valores chegam a 85,23\% a uma profundidade de $60 \mathrm{~cm}$, o que classifica o solo como sódico. Resultados 
Quadro 2. Resultado das medisas descritivas de $\mathrm{pH}$ no solo e CE no extrato da pasta de saturação de solos do Perímetro Irrigado Cachoeira II, Serra Talhada - PE.

\begin{tabular}{|c|c|c|c|c|c|c|c|c|}
\hline & \multicolumn{4}{|c|}{ pH do solo (água 1:2,5) } & \multicolumn{4}{|c|}{$\mathrm{CE}\left(\mathrm{dS} \mathrm{m}^{-1}\right)$} \\
\hline & jun & set & dez & mar & jun & set & dez & mar \\
\hline \multicolumn{9}{|c|}{$0-20 \mathrm{~cm}$} \\
\hline Média & 8,44 & 7,86 & 7,93 & 8,02 & 2,72 & 2,42 & 3,13 & 2,25 \\
\hline Mediana & 8,50 & 8,00 & 7,90 & 8,00 & 0,78 & 0,99 & 1,17 & 0,66 \\
\hline Moda & 8,00 & 8,00 & 7,90 & 8,20 & 0,78 & 0,85 & 0,95 & 0,31 \\
\hline $\mathrm{DP}^{7}$ & 0,67 & 0,56 & 0,42 & 0,56 & 7,59 & 5,43 & 7,90 & 4,33 \\
\hline $\mathrm{CV}^{8}$ & 7,89 & 7,12 & 5,23 & 7,01 & 279,02 & 224,38 & 252,58 & 192,77 \\
\hline Curtose & $-0,87$ & 0,18 & $-0,90$ & 0,22 & 16,32 & 15,63 & 23,18 & 8,46 \\
\hline Máxima & 9,60 & 8,70 & 8,70 & 9,50 & 37,00 & 26,70 & 44,70 & 19,46 \\
\hline Mínima & 7,20 & 6,50 & 7,30 & 6,90 & 0,37 & 0,16 & 0,40 & 0,25 \\
\hline \multicolumn{9}{|c|}{$20-40 \mathrm{~cm}$} \\
\hline Média & 9,15 & 8,23 & 8,24 & 8,28 & 1,83 & 1,74 & 2,08 & 2,51 \\
\hline Mediana & 9,20 & 8,30 & 8,20 & 8,30 & 0,76 & 0,77 & 0,93 & 0,58 \\
\hline Moda & 9,60 & 8,50 & 8,60 & 7,80 & 0,82 & 0,59 & 1,16 & 0,49 \\
\hline $\mathrm{DP}^{7}$ & 0,63 & 0,53 & 0,49 & 0,47 & 4,06 & 3,38 & 5,26 & 4,61 \\
\hline $\mathrm{CV}^{8}$ & 6,90 & 6,46 & 5,99 & 5,66 & 221,87 & 194,11 & 253,33 & 183,67 \\
\hline Curtose & $-0,17$ & 0,86 & 3,18 & $-0,30$ & 17,26 & 18,16 & 30,60 & 7,05 \\
\hline Máxima & 10,20 & 9,00 & 9,22 & 9,40 & 21,10 & 18,30 & 31,88 & 18,67 \\
\hline Mínima & 7,60 & 6,70 & 6,50 & 7,40 & 0,26 & 0,08 & 0,18 & 0,24 \\
\hline \multicolumn{9}{|c|}{$40-60 \mathrm{~cm}$} \\
\hline Média & 9,57 & 8,32 & 8,42 & 8,44 & 1,68 & 1,80 & 2,56 & 2,57 \\
\hline Mediana & 9,60 & 8,40 & 8,40 & 8,40 & 0,81 & 0,82 & 0,92 & 0,89 \\
\hline Moda & 9,50 & 8,40 & 8,10 & 8,00 & 0,37 & 0,70 & 0,64 & 0,28 \\
\hline $\mathrm{DP}^{7}$ & 0,62 & 0,53 & 0,55 & 0,54 & 3,42 & 4,02 & 4,88 & 4,27 \\
\hline $\mathrm{CV}^{8}$ & 6,51 & 6,42 & 6,59 & 6,41 & 203,46 & 222,88 & 190,70 & 166,11 \\
\hline Curtose & $-1,00$ & 0,69 & $-0,52$ & $-0,09$ & 19,50 & 25,11 & 17,27 & 10,34 \\
\hline Máxima & 10,70 & 9,20 & 9,50 & 9,80 & 18,90 & 23,30 & 26,80 & 21,20 \\
\hline Mínima & 8,50 & 6,80 & 7,30 & 7,50 & 0,28 & 0,06 & 0,18 & 0,26 \\
\hline
\end{tabular}

semelhantes foram encontrados por Chaves et al. (2006) na Ilha de Picos - PE.

Verificou-se, também, acúmulo progressivo de sódio no solo, com maiores valores na estação seca do ano, promovido pela ascensão capilar dos sais devido ao processo de evaporação, demonstrando que futuramente poderá haver problemas de sodificação nos solos do perímetro. Como forma de impedir o aumento no acúmulo destes sais, a subárea afetada deve ser isolada da normal, pela construção de diques (PIZARRO, 1978; MEDEIROS; GHEYI, 1997; PESSOA, 2012). Na realização de pesquisas, procedimentos diferenciados devem ser adotados levando-se em consideração a subdivisão da área e sua variabilidade espacial.

Os valores do CV para sódio e PST, segundo Warrick e Nielsen (1980), foram altos, concordando com o que foi constatado por Souza, Cogo e Vieira (1997) e Silva (2001). A variabilidade dessas propriedades pode ser atribuída, em parte, aos processos de formação do solo aluvial, além da evolução da sodicidade pelo uso de águas de irrigação de diferentes teores de sais de sódio, bem como do manejo adotado na irrigação. De acordo com Santos e Vasconcelos (1987), Souza, Cogo e Vieira (1997), Salviano, Vieira e Sparovek (1998), Oliveira et al. (1999) e Silva (2001), o conhecimento da variabilidade dessa propriedade é importante, principalmente para definir o manejo mais adequado a ser utilizado na área em estudo.

No Quadro 4, estão expressos os resultados das concentrações de cátions na solução do solo nos lotes do Perímetro. Verifica-se que existe uma grande amplitude de variação para os cátions solúveis, o que acarreta um CV elevado, resultado da heterogeneidade dos solos da área, principalmente no que se refere ao acúmulo de sais.

Isso é observado para os cátions solúveis $\mathrm{Ca}^{2+}, \mathrm{Mg}^{2+}$, $\mathrm{Na}^{+}$e $\mathrm{K}^{+}$, refletindo-se também na RAS desses solos, fato que se repete nas quatro épocas de coleta. É possível que a 
Quadro 3. Medidas descritivas dos teores de cátions trocáveis $\left(\mathrm{cmol}_{\mathrm{c}} \mathrm{dm}^{-3}\right)$ nos solos do Perímetro Irrigado Cachoeira II, Serra Talhada - PE.

\begin{tabular}{|c|c|c|c|c|c|c|c|c|c|c|c|c|c|c|c|}
\hline \multirow{2}{*}{ Medidas } & $\mathrm{Ca}$ & Mg & $\mathrm{Na}$ & $\mathbf{K}$ & PST & $\mathrm{Ca}$ & Mg & $\mathrm{Na}$ & $\mathbf{K}$ & PST & $\mathbf{C a}$ & Mg & $\mathrm{Na}$ & $\mathbf{K}$ & PST \\
\hline & \multicolumn{5}{|c|}{$0-20 \mathrm{~cm}$} & \multicolumn{5}{|c|}{$20-40 \mathrm{~cm}$} & \multicolumn{5}{|c|}{$40-60 \mathrm{~cm}$} \\
\hline \multicolumn{16}{|c|}{ junho } \\
\hline Média & 8,09 & 2,83 & 1,97 & 1,14 & 10,11 & 8,15 & 2,75 & 2,00 & 0,70 & 12,69 & 8,43 & 2,79 & 2,91 & 0,70 & 16,00 \\
\hline Mediana & 8,01 & 3,05 & 0,75 & 0,72 & 6,38 & 7,98 & 2,96 & 1,30 & 0,56 & 9,58 & 8,46 & 3,03 & 1,43 & 0,48 & 11,84 \\
\hline Moda & ne & 3,15 & 0,61 & 0,64 & ne & ne & 2,28 & 0,61 & 0,32 & ne & ne & ne & 1,02 & 0,48 & ne \\
\hline $\mathrm{DP}$ & 1,37 & 0,55 & 4,46 & 1,00 & 13,37 & 1,83 & 0,65 & 2,90 & 0,64 & 13,44 & 1,87 & 0,68 & 4,54 & 0,52 & 15,04 \\
\hline $\mathrm{CV}$ & 16,88 & 19,48 & 227,04 & 87,96 & 132,22 & 22,45 & 23,85 & 145,16 & 91,22 & 105,89 & 22,20 & 24,49 & 156,05 & 73,18 & 94,00 \\
\hline Curtose & $-0,37$ & 3,63 & 19,50 & 4,74 & 12,57 & $-0,12$ & 1,11 & 13,50 & 9,01 & 13,17 & 0,11 & 0,73 & 20,51 & 4,97 & 12,59 \\
\hline $\operatorname{Max}$ & 11,02 & 3,54 & 24,22 & 4,87 & 64,11 & 11,40 & 3,57 & 15,42 & 3,16 & 75,23 & 11,59 & 3,60 & 26,35 & 2,68 & 85,23 \\
\hline Min & 5,24 & 0,84 & 0,20 & 0,24 & 1,75 & 3,66 & 0,92 & 0,20 & 0,24 & 1,76 & 3,33 & 0,83 & 0,20 & 0,24 & 2,06 \\
\hline \multicolumn{16}{|c|}{ setembro } \\
\hline Média & 6,20 & 5,38 & 2,76 & 1,39 & 9,52 & 6,83 & 5,11 & 2,36 & 0,78 & 11,03 & 7,51 & 5,44 & 3,21 & 0,52 & 13,76 \\
\hline Mediana & 5,56 & 5,01 & 0,73 & 1,28 & 6,43 & 6,40 & 5,42 & 1,08 & 0,72 & 7,71 & 7,08 & 5,16 & 1,57 & 0,44 & 9,63 \\
\hline Moda & ne & ne & 0,20 & 1,58 & ne & ne & 5,47 & 1,08 & 0,17 & ne & ne & 3,43 & 1,17 & 0,12 & ne \\
\hline $\mathrm{DP}$ & 1,71 & 2,29 & 8,04 & 0,66 & 13,54 & 2,08 & 1,06 & 5,44 & 0,46 & 14,63 & 2,74 & 1,50 & 6,93 & 0,35 & 15,82 \\
\hline $\mathrm{CV}$ & 27,65 & 42,60 & 291,50 & 47,86 & 142,22 & 30,50 & 20,70 & 230,70 & 58,18 & 132,71 & 36,46 & 27,57 & 216,07 & 67,70 & 114,91 \\
\hline Curtose & 1,33 & 17,53 & 19,18 & 2,06 & 11,48 & $-1,02$ & $-0,31$ & 20,75 & $-0,65$ & 12,49 & $-0,71$ & $-0,49$ & 23,76 & 1,81 & 11,74 \\
\hline $\operatorname{Max}$ & 11,58 & 16,83 & 42,45 & 3,55 & 64,28 & 10,63 & 7,62 & 30,16 & 1,83 & 76,70 & 13,27 & 8,87 & 39,82 & 1,58 & 84,56 \\
\hline Min & 3,82 & 2,77 & 0,10 & 0,27 & 0,78 & 3,53 & 3,19 & 0,10 & 0,12 & 1,12 & 2,91 & 3,41 & 0,10 & 0,12 & 1,14 \\
\hline \multicolumn{16}{|c|}{ dezembro } \\
\hline Média & 10,62 & 12,47 & 3,76 & 1,33 & 8,81 & 11,44 & 13,01 & 3,44 & 0,66 & 9,53 & 12,27 & 14,12 & 5,07 & 0,51 & 13,30 \\
\hline Mediana & 9,85 & 11,14 & 1,61 & 1,28 & 5,58 & 11,12 & 12,12 & 1,89 & 0,62 & 6,41 & 11,92 & 12,72 & 3,51 & 0,46 & 9,26 \\
\hline Moda & ne & 12,58 & 1,00 & 1,51 & ne & ne & ne & 3,28 & 0,46 & ne & ne & ne & 1,42 & 0,19 & ne \\
\hline $\mathrm{DP}$ & 3,25 & 4,78 & 9,90 & 0,60 & 11,77 & 4,29 & 5,52 & 6,42 & 0,36 & 11,83 & 5,22 & 5,32 & 6,60 & 0,32 & 13,08 \\
\hline $\mathrm{CV}$ & 30,60 & 38,33 & 263,36 & 44,74 & 133,68 & 37,50 & 42,44 & 186,59 & 54,27 & 124,11 & 42,58 & 37,68 & 130,18 & 62,59 & 98,33 \\
\hline Curtose & $-1,19$ & 1,62 & 25,07 & $-0,84$ & 15,21 & $-0,46$ & 2,00 & 20,88 & 3,50 & 23,54 & 0,41 & $-0,79$ & 13,91 & 9,84 & 11,49 \\
\hline $\operatorname{Max}$ & 16,94 & 26,88 & 57,02 & 2,55 & 63,54 & 20,59 & 30,16 & 36,40 & 1,88 & 72,56 & 25,97 & 24,53 & 36,40 & 1,93 & 72,70 \\
\hline Min & 5,80 & 6,51 & 0,00 & 0,41 & 0,00 & 3,86 & 5,09 & 0,22 & 0,24 & 1,49 & 2,64 & 5,91 & 0,22 & 0,19 & 1,34 \\
\hline \multicolumn{16}{|c|}{ março } \\
\hline Média & 7,00 & 2,94 & 0,62 & 0,36 & 5,63 & 6,61 & 3,20 & 0,93 & 0,24 & 8,16 & 7,32 & 3,42 & 1,19 & 0.19 & 9,59 \\
\hline Mediana & 6,90 & 3,00 & 0,38 & 0,30 & 3,16 & 6,92 & 2,96 & 0,48 & 0,20 & 5,16 & 7,65 & 3,31 & 0,91 & 0,16 & 7,66 \\
\hline Moda & 8,48 & 2,44 & 0,50 & 0,47 & ne & ne & 2,38 & 0,29 & 0,11 & ne & ne & ne & 0,91 & 0,22 & ne \\
\hline DP & 2,04 & 0,65 & 0,66 & 0,19 & 5,67 & 1,95 & 1,02 & 1,05 & 0,15 & 9,41 & 2,41 & 1,03 & 1,11 & 0,11 & 8,67 \\
\hline $\mathrm{CV}$ & 29,11 & 22,02 & 106,55 & 53,55 & 100,74 & 29,47 & 31,84 & 112,61 & 63,28 & 115,33 & 32,86 & 30,19 & 93,22 & 57,86 & 90,39 \\
\hline Curtose & $-0,55$ & 2,21 & 4,73 & $-0,31$ & 4,22 & $-0,47$ & 1,73 & 4,64 & 3,76 & 10,11 & 0,12 & 0,70 & 6,41 & 4,19 & 9,71 \\
\hline $\operatorname{Max}$ & 10,95 & 4,99 & 3,10 & 0,85 & 24,19 & 9,87 & 6,47 & 4,52 & 0,77 & 44,95 & 13,73 & 6,45 & 5,33 & 0,55 & 44,68 \\
\hline Min & 2,51 & 1,68 & 0,11 & 0,12 & 0,86 & 2,10 & 2,00 & 0,15 & 0,08 & 1,33 & 2,96 & 1,70 & 0,16 & 0,08 & 1,56 \\
\hline
\end{tabular}

disponibilidade hídrica e a qualidade das águas utilizadas no Perímetro estejam influenciando essa heterogeneidade, em virtude das diferentes classes de água disponíveis, variáveis no tempo e no espaço. Resultados semelhantes foram relatados por Herrero e Pérez-Coveta (2005) em trabalho realizado em um perímetro irrigado com problemas de salinização no sul da Espanha. Segundo Rhoades (1974), dentre os principais fatores que causam a salinização, a qualidade da água de irrigação contribui efetivamente para aumentar o teor de sais na solução do solo, pois ela pode conter de $100 \mathrm{~kg}$ (água boa) a $4.000 \mathrm{~kg}$ (água imprópria) de sal em cada $1.000 \mathrm{~m}^{3}$ e é, em geral, aplicada à razão de $10.000 \mathrm{a} 15.000 \mathrm{~m}^{3} \mathrm{ha}^{-1} \mathrm{ano}^{-1}$. Consequentemente, se não houver drenagem, cerca de 1,0 a 60,0 t há ${ }^{-1}$ de sais poderão ser adicionadas às áreas irrigadas. 
Quadro 4. Medidas descritivas dos teores de cátions solúveis $\left(\mathrm{mmol}_{\mathrm{c}} \mathrm{L}^{-1}\right)$ nos solos do Perímetro Irrigado Cachoeira II, Serra Talhada - PE.

\begin{tabular}{|c|c|c|c|c|c|c|c|c|c|c|c|c|c|c|c|}
\hline \multirow{2}{*}{ Medidas } & $\mathbf{C a}$ & Mg & $\mathrm{Na}$ & $\mathbf{K}$ & PST & Ca & Mg & $\mathbf{N a}$ & $\mathbf{K}$ & PST & $\mathbf{C a}$ & Mg & $\mathrm{Na}$ & $\mathbf{K}$ & PST \\
\hline & \multicolumn{5}{|c|}{$0-20 \mathrm{~cm}$} & \multicolumn{5}{|c|}{$20-40 \mathrm{~cm}$} & \multicolumn{5}{|c|}{$40-60 \mathrm{~cm}$} \\
\hline \multicolumn{16}{|c|}{ junho } \\
\hline Média & 0,77 & 0,77 & 2,07 & 0,08 & 1,27 & 0,47 & 0,45 & 1,11 & 0,03 & 1,23 & 0,35 & 0,32 & 1,30 & 0,02 & 1,59 \\
\hline Mediana & 0,35 & 0,37 & 0,26 & 0,05 & 0,45 & 0,24 & 0,23 & 0,36 & 0,02 & 0,80 & 0,18 & 0,20 & 0,37 & 0,02 & 0,99 \\
\hline Moda & ne & $\mathrm{Ne}$ & 0,22 & 0,02 & ne & 0,18 & ne & 0,13 & 0,02 & ne & ne & 0,19 & ne & 0,01 & ne \\
\hline DP & 1,42 & 1,40 & 7,67 & 0,09 & 3,42 & 0,72 & 0,64 & 3,10 & 0,03 & 1,90 & 0,52 & 0,38 & 4,32 & 0,01 & 3,18 \\
\hline $\mathrm{CV}$ & 183,85 & 182,76 & 370,55 & 113,30 & 269,60 & 154,59 & 143,21 & 278,31 & 97,04 & 154,94 & 154,94 & 121,67 & 332,77 & 54,91 & 200,22 \\
\hline Curtose & 17,46 & 27,99 & 18,72 & 14,22 & 16,31 & 17,05 & 12,09 & 26,82 & 7,91 & 26,01 & 9,77 & 7,77 & 34,17 & $-0,01$ & 33,23 \\
\hline Max & 7,73 & 8,54 & 39,42 & 0,50 & 16,68 & 4,00 & 3,11 & 18,12 & 0,15 & 11,60 & 2,47 & 1,65 & 26,38 & 0,06 & 19,96 \\
\hline Min & 0,12 & 0,12 & 0,04 & 0,01 & 0,04 & 0,08 & 0,05 & 0,05 & 0,01 & 0,11 & 0,08 & 0,05 & 0,05 & 0,01 & 0,10 \\
\hline \multicolumn{16}{|c|}{ setembro } \\
\hline Média & 0,35 & 0,16 & 2,02 & 0,10 & 3,32 & 0,23 & 0,14 & 1,61 & 0,04 & 3,60 & 0,22 & 0,12 & 1,26 & 0,03 & 3,54 \\
\hline Mediana & 0,14 & 0,08 & 0,41 & 0,08 & 1,89 & 0,10 & 0,08 & 0,41 & 0,03 & 2,06 & 0,09 & 0,07 & 0,51 & 0,02 & 2,63 \\
\hline Moda & ne & ne & 0,39 & 0,03 & ne & ne & ne & 0,21 & 0,05 & ne & ne & ne & 0,70 & 0,01 & ne \\
\hline DP & 0,87 & 0,35 & 6,68 & 0,10 & 5,50 & 0,48 & 0,25 & 5,28 & 0,04 & 5,65 & 0,50 & 0,22 & 3,14 & 0,03 & 3,60 \\
\hline $\mathrm{CV}$ & 247,25 & 214,41 & 329,99 & 101,37 & 165,86 & 211,42 & 179,28 & 328,55 & 93,45 & 156,93 & 225,86 & 195,13 & 249,32 & 94,44 & 101,61 \\
\hline Curtose & 15,46 & 15,66 & 23,46 & 11,29 & 20,40 & 18,09 & 18,89 & 32,15 & 3,83 & 19,03 & 14,62 & 27,30 & 27,42 & 0,78 & 10,43 \\
\hline $\operatorname{Max}$ & 3,95 & 1,64 & 37,28 & 0,58 & 31,52 & 2,54 & 1,38 & 31,87 & 0,19 & 32,23 & 2,25 & 1,34 & 18,43 & 0,11 & 19,47 \\
\hline Min & 0,03 & 0,04 & 0,01 & 0,01 & 0,09 & 0,02 & 0,01 & 0,02 & 0,01 & 0,17 & 0,02 & 0,03 & 0,02 & 0,01 & 0,13 \\
\hline \multicolumn{16}{|c|}{ dezembro } \\
\hline Média & 0,21 & 0,03 & 6,40 & 0,08 & 9,01 & 0,06 & 0,02 & 3,98 & 0,03 & 13,34 & 0,07 & 0,04 & 5,49 & 0,03 & 25,77 \\
\hline Mediana & 0,07 & 0,02 & 0,84 & 0,07 & 3,25 & 0,04 & 0,01 & 0,87 & 0,02 & 4,64 & 0,03 & 0,01 & 1,47 & 0,01 & 8,19 \\
\hline Moda & ne & 0,02 & 0,84 & 0,01 & ne & ne & 0,01 & 1,08 & 0,01 & ne & ne & 0,03 & 0,80 & 0,01 & ne \\
\hline DP & 0,51 & 0,04 & 25,75 & 0,06 & 21,66 & 0,07 & 0,02 & 14,11 & 0,05 & 33,83 & 0,16 & 0,10 & 15,43 & 0,04 & 66,02 \\
\hline $\mathrm{CV}$ & 240,18 & 117,37 & 402,67 & 70,96 & 240,27 & 114,76 & 101,77 & 354,46 & 141,07 & 253,52 & 227,93 & 275,35 & 281,08 & 143,87 & 256,18 \\
\hline Curtose & 19.96 & 14,16 & 28,73 & $-0,20$ & 24,57 & 2,10 & 2,68 & 27,80 & 17,44 & 27,03 & 22,99 & 34,75 & 24,04 & 13,39 & 26,66 \\
\hline $\operatorname{Max}$ & 2,77 & 0,21 & 149,89 & 0,23 & 125,07 & 0,28 & 0,08 & 81,94 & 0,27 & 199,14 & 0,89 & 0,61 & 88,12 & 0,22 & 388,16 \\
\hline Min & 0,00 & 0,00 & 0,11 & 0,01 & 0,59 & 0,00 & 0,00 & 0,14 & 0,01 & 0,68 & 0,00 & 0,00 & 0,26 & 0,01 & 0,48 \\
\hline \multicolumn{16}{|c|}{ março } \\
\hline Média & 0,16 & 0,19 & 1,07 & 0,05 & 2,97 & 0,16 & 0,19 & 1,22 & 0,04 & 3,79 & 0,13 & 0,15 & 1,40 & 0,04 & 5,04 \\
\hline Mediana & 0,07 & 0,11 & 0,19 & 0,04 & 1,17 & 0,05 & 0,08 & 0,23 & 0,03 & 1,53 & 0,05 & 0,08 & 0,36 & 0,03 & 1,89 \\
\hline Moda & ne & 0,07 & 0,14 & 0,03 & ne & ne & ne & 0,18 & 0,03 & ne & 0,02 & ne & 0,16 & 0,03 & ne \\
\hline $\mathrm{DP}$ & 0,25 & 0,24 & 2,46 & 0,04 & 6,18 & 0,28 & 0,29 & 2,49 & 0,02 & 7,27 & 0,23 & 0,20 & 2,59 & 0,03 & 8,60 \\
\hline $\mathrm{CV}$ & 156,50 & 126,80 & 228,95 & 67,89 & 208,29 & 175,91 & 157,39 & 204,12 & 39,74 & 191,86 & 176,37 & 138,72 & 185,83 & 76,64 & 170,62 \\
\hline Curtose & 7,96 & 11,10 & 7,10 & 11,48 & 14,53 & 8,28 & 10,56 & 6,51 & 4,90 & 15,31 & 9,75 & 13,45 & 6,83 & 12,18 & 12,84 \\
\hline Max & 1,10 & 1,24 & 9,64 & 0,22 & 31,85 & 1,26 & 1,41 & 10,31 & 0,09 & 38,11 & 1,12 & 1,07 & 11,09 & 0,20 & 44,22 \\
\hline Min & 0,02 & 0,04 & 0,07 & 0,03 & 0,34 & 0,01 & 0,03 & 0,09 & 0,01 & 0,46 & 0,01 & 0,03 & 0,11 & 0,01 & 0,75 \\
\hline
\end{tabular}

Essa é uma quantidade expressiva de sais e pode causar sérios danos ao uso do solo para o desenvolvimento da atividade agrícola, conforme observado por Sousa (1995).

A variação temporal da concentração de sódio no extrato de saturação do solo, entre as coletas está representada no Quadro 4. Observou-se, de forma geral, um comportamento bastante variável nos teores do referido íon durante o período estudado. No perímetro irrigado, a maior concentração na primeira camada foi registrada no mês de dezembro (Coleta 3 ), $6,40 \mathrm{mmol}_{\mathrm{c}} \mathrm{L}^{-1}$. Este resultado corrobora os apresentados por Andrade et al. (2002) em seus estudos com a concentração de íons no extrato de saturação do solo, na região da Chapada do Apodi. Tal comportamento está associado à ausência de chuvas nesse período, o que pode estar ocasionando um aumento 
na concentração de sódio na solução do solo em resposta à qualidade da água empregada, ao manejo de irrigação adotado e também à elevada evapotranspiração da época. Nas camadas de 20-40 e 40-60 cm, observou-se, a mesma tendência da camada superficial, apresentando os maiores valores de 3,98 e 5,49 $\mathrm{mmol}_{\mathrm{c}} \mathrm{L}^{-1}$, respectivamente para cada camada na coleta 3 para o íon sódio.

Tais resultados concordam com a afirmativa de Ayers e Westcot (1999) de que, à medida que a cultura extrai água da zona radicular e o solo se torna seco entre as irrigações, os íons tendem a se acumular nas camadas inferiores do solo. Contudo, se a evapotranspiração for elevada, poderá ocorrer ascensão capilar de íons e concentração na camada superficial do solo.

As maiores concentrações do íon sódio em forma solúvel na estação seca foram encontradas na camada superior, em decorrência da perda de água por evaporação. Contudo, isso permanece no período chuvoso, podendo indicar maiores aportes desse elemento em detrimento dos demais cátions, o que poderá promover a sodificação dos solos com o tempo (FREIRE et al., 2003b).

Semelhante tendência foi observada por Pereira, Matias Filho e Andrade (1986) e Ben-Hur et al. (2001), quando estudaram a ação da lixiviação dos sais adicionados ao solo pela água de irrigação em solos de textura leve, em que as maiores concentrações de sódio foram encontradas nas camadas superficiais em solos com problemas na lixiviação.

As concentrações médias de $\mathrm{Ca}^{2+}$ e $\mathrm{Mg}^{2+}$ também estão representadas no Quadro 4. Os valores das concentrações dos referidos íons variaram de 0,16 a 0,77 e 0,03 a $0,77 \mathrm{mmol}_{\mathrm{c}} \mathrm{L}^{-1}$, na primeira camada; 0,6 a 0,47 e 0,02 a $0,45 \mathrm{mmol}_{\mathrm{c}} \mathrm{L}^{-1}$, na segunda camada; e 0,07 a 0,35 e 0,04 a 0,32 $\mathrm{mmol}_{\mathrm{c}} \mathrm{L}^{-1}$, na terceira camada, respectivamente. $\mathrm{Na}$ camada superficial, observa-se um decréscimo da concentração dos íons $\mathrm{Ca}^{2+} \mathrm{e}$ $\mathrm{Mg}^{2+}$ durante a estação seca da região (Coleta 3).

Esse comportamento difere do encontrado por Andrade et al. (2004) nos campos irrigados da Chapada do Apodi. Acredita-se que esse comportamento seja devido às características intrínsecas do solo de cada região, pois os solos do Perímetro Irrigado Cachoeira II possuem deposições sucessivas de camadas promovidas pelas enchentes do Rio Pajeú, fato que não ocorre na Chapada do Apodi.

As concentrações de potássio encontradas foram relativamente baixas entre as coletas. Estas baixas concentrações do íon podem ser atribuídas ao fato de que o potássio é perdido por lixiviação e altamente consumido pelas culturas exploradas no perímetro, a exemplo da bananeira.

Os solos afetados por sais são classificados em função dos valores do pHes, CEes e PST (VAN RAIJ, 1991). Neste contexto e para fins de caracterização da salinidade e sodicidade na área estudada, foram tomados como base os valores dessas variáveis e a classificação de solos apresentada por Richards (1954). Verifica-se que aproximadamente $12 \%, 14 \%$ e $28 \%$ da área estudada estão afetados por sais, nas profundidades de 0-20, 20-40 e 40-60 cm, respectivamente. Resultados semelhantes foram obtidos por Coelho e Ferreyra (1986) em Neossolo Flúvico (solo aluvial) no Perímetro Irrigado de Morada Nova - CE; Souza, Queiroz e Gheyi (2000) no Semiárido Paraibano; e por Chaves (2004) na Ilha de Assunção, em Cabrobó - PE.
Quanto aos ânions avaliados, em geral, os valores de $\mathrm{CO}_{3} \mathrm{e}$ $\mathrm{HCO}_{3}$ na solução do solo são baixos e seguem a tendência de baixar ainda mais em profundidade (Quadro 5). A concentração desses ânions não caracteriza problemas ao solo estudado, nem às culturas exploradas comercialmente no perímetro, sendo os valores de $\mathrm{CO}_{3}{ }^{2-}$ tão baixos que se encontram fora da faixa de detecção do método utilizado na segunda e terceira coletas. Comparativamente, essas informações podem ser contrastadas e confirmadas no trabalho realizado por Corwin et al. (2006), avaliando as características e a qualidade do solo por intermédio do monitoramento do manejo do solo, observando o decréscimo nos valores de $\mathrm{CO}_{3}{ }^{2-}$ e $\mathrm{HCO}_{3}{ }^{-}$em algumas condições de manejo.

Observa-se, ainda, $\mathrm{CV}$ médio para $\mathrm{HCO}_{3}^{-}$nas três profundidades e quatro amostragens realizadas, enquanto para $\mathrm{CO}_{3}{ }^{2-}$ apresenta $\mathrm{CV}$ elevado em virtude da não detecção do $\mathrm{CO}_{3}{ }^{2-}$ pela metodologia adotada.

O ânion que predomina nos solos do Perímetro é o $\mathrm{Cl}^{-}$, destacando-se dos demais. A distribuição do íon cloreto no extrato de saturação da coleta 3 (dezembro de 2006), nas três camadas, foi a que apresentou maior valor médio entre as coletas, sendo de $6,22 \mathrm{mmol} . \mathrm{L}^{-1} ; 3,64$ e $4,77 \mathrm{mmol} . \mathrm{L}^{-1}$, respectivamente, para as profundidades de 0-20, 20-40 e 40-60 cm. Esse acréscimo nos valores com relação às outras coletas deve-se à época da amostragem ter sido no final do período seco, fase de aumento na concentração de sais pela elevada evapotranspiração e nula precipitação durante meses, o que é confirmado pela CE nas amostras de solos dessa coleta (Quadro 2).

Os altos teores de $\mathrm{Cl}^{-}$encontrados nos solos coletados em dezembro de 2006 (terceira coleta) decrescem novamente na quarta e última coleta, em março de 2007, pois, nesse período, já haviam ocorrido chuvas, diluindo os sais na solução do solo.

Esse comportamento concorda com os verificados por Pérez-Sirvent et al. (2003) no semiárido no sul da Espanha e por Andrade et al. (2004) no perímetro irrigado do DIJA (Distrito Irrigado Jaguaribe Apodi), no Ceará. Os altos valores do cloreto registrados na área de perímetro podem ser explicados pela utilização de águas subterrâneas (C3S1), cloretadas, na irrigação e pelo resíduo do cloreto de potássio empregado na fertirrigação como fonte de potássio para as culturas, especialmente a bananeira, bastante exigente em potássio (Andrade et al., 2002).

O elevado CV observado pode ser atribuído a três fatores distintos, sendo o primeiro a qualidade da água. Parte do perímetro é irrigada com águas superficiais que apresentam menores concentrações de sais totais, sendo classificadas como C2S1 (CRUZ, 2001). O segundo fator é a alteração no manejo adotado, ou seja, irrigação por sulco em algumas áreas e irrigação por aspersão, ou microaspersão em outras. E, por último, o tempo em que a área vem sendo cultivada. Alguns lotes não estão sendo irrigados há alguns anos, o que reduz o aporte de sais nessas áreas. Resultados semelhantes foram apresentados por Ben-Hur et al. (2001), nos estudos com solos irrigados nos Estados Unidos.

Todavia, as concentrações de cloreto registradas no período de estudo (junho de 2006 a março de 2007), superaram os limites médios de toxidez apresentados por Ayers e Westcot (1999), que são de 5 a $10 \mathrm{mmol}_{\mathrm{c}} \mathrm{L}^{-1}$, apenas na camada 
Quadro 5. Resultado das medidas descritivas dos ânions $\left(\mathrm{mmol}_{\mathrm{c}} \mathrm{L}^{-1}\right)$ no Perímetro Irrigado Cachoeira II, Serra Talhada -PE.

\begin{tabular}{|c|c|c|c|c|c|c|c|c|c|}
\hline \multirow{2}{*}{ Medidas } & $\mathrm{Cl}^{-}$ & $\mathrm{HCO}_{3}{ }^{2-}$ & $\mathrm{CO}_{3}$ & $\mathrm{Cl}^{-}$ & $\mathrm{HCO}_{3}{ }^{2-}$ & $\mathrm{CO}_{3}$ & $\mathrm{Cl}^{-}$ & $\mathrm{HCO}_{3}{ }^{2-}$ & $\mathrm{CO}_{3}$ \\
\hline & \multicolumn{3}{|c|}{$0-20 \mathrm{~cm}$} & \multicolumn{3}{|c|}{$20-40 \mathrm{~cm}$} & \multicolumn{3}{|c|}{$40-60 \mathrm{~cm}$} \\
\hline \multicolumn{10}{|c|}{ junho } \\
\hline Média & 3,26 & 0,41 & 0,03 & 1,80 & 0,37 & 0,03 & 1,86 & 0,34 & 0,02 \\
\hline Mediana & 0,85 & 0,40 & 0,05 & 0,65 & 0,30 & 0,00 & 0,80 & 0,30 & 0,00 \\
\hline Moda & 0,85 & 0,40 & 0,05 & 0,30 & 0,30 & 0,00 & 0,30 & 0,40 & 0,00 \\
\hline DP & 10,16 & 0,15 & 0,03 & 4,25 & 0,12 & 0,03 & 4,83 & 0,12 & 0,02 \\
\hline $\mathrm{CV}$ & 311,17 & 36,12 & 89,10 & 236,42 & 32,99 & 110,55 & 258,83 & 34,70 & 124,48 \\
\hline Curtose & 20,91 & 0,00 & $-0,88$ & 19,40 & $-0,42$ & $-0,72$ & 29,68 & 0,58 & $-1,86$ \\
\hline Max & 55,00 & 0,80 & 0,10 & 23,00 & 0,60 & 0,10 & 29,00 & 0,70 & 0,05 \\
\hline Min & 0,25 & 0,20 & 0,00 & 0,20 & 0,20 & 0,00 & 0,20 & 0,20 & 0,00 \\
\hline \multicolumn{10}{|c|}{ setembro } \\
\hline Média & 2,08 & 1,06 & 0,00 & 1,44 & 1,03 & 0,00 & 1,09 & 0,96 & 0,00 \\
\hline Mediana & 0,22 & 1,00 & 0,00 & 0,18 & 1,00 & 0,00 & 0,22 & 1,00 & 0,00 \\
\hline Moda & 0,17 & 1,00 & 0,00 & 0,25 & 1,00 & 0,00 & 0,10 & 1,00 & 0,00 \\
\hline DP & 7,80 & 0,19 & 0,00 & 5,83 & 0,33 & 0,00 & 3,72 & 0,28 & 0,00 \\
\hline $\mathrm{CV}$ & 374,65 & 18,27 & ne & 405,27 & 31,87 & ne & 342,24 & 28,90 & ne \\
\hline Curtose & 21,34 & 1,28 & ne & 32,77 & 3,23 & ne & 25,37 & 1,70 & ne \\
\hline $\operatorname{Max}$ & 42,00 & 1,50 & 0,00 & 35,00 & 2,10 & 0,00 & 21,00 & 1,70 & 0,00 \\
\hline Min & 0,04 & 0,70 & 0,00 & 0,02 & 0,20 & 0,00 & 0,01 & 0,30 & 0,00 \\
\hline \multicolumn{10}{|c|}{ dezembro } \\
\hline Média & 6,22 & 1,21 & 0,00 & 3,64 & 1,06 & 0,00 & 4,77 & 1,13 & 0,00 \\
\hline Mediana & 0,60 & 1,00 & 0,00 & 0,54 & 1,00 & 0,00 & 0,58 & 1,00 & 0,00 \\
\hline Moda & 1,00 & 1,00 & 0,00 & 0,42 & 1,00 & 0,00 & 0,12 & 1,00 & 0,00 \\
\hline $\mathrm{DP}$ & 25,89 & 0,70 & 0,00 & 13,85 & 0,32 & 0,00 & 14,94 & 0,53 & 0,00 \\
\hline $\mathrm{CV}$ & 416,13 & 58,33 & ne & 380,93 & 30,00 & ne & 313,43 & 46,61 & ne \\
\hline Curtose & 28,39 & 14,16 & ne & 27,56 & 11,42 & ne & 24,37 & 10,95 & ne \\
\hline Max & 150,00 & 4,50 & 0,00 & 80,00 & 2,50 & 0,00 & 85,00 & 3,50 & 0,00 \\
\hline Min & 0,09 & 0,30 & 0,00 & 0,02 & 0,60 & 0,00 & 0,06 & 0,60 & 0,00 \\
\hline \multicolumn{10}{|c|}{ março } \\
\hline Média & 1,61 & 0,01 & 0,02 & 1,82 & 0,01 & 0,01 & 1,90 & 0,01 & 0,01 \\
\hline Mediana & 0,60 & 0,01 & 0,00 & 1,00 & 0,01 & 0,00 & 1,00 & 0,01 & 0,00 \\
\hline Moda & 1,00 & 0,01 & 0,00 & 1,00 & 0,01 & 0,00 & 1,00 & 0,01 & 0,00 \\
\hline DP & 2,56 & 0,00 & 0,05 & 2,69 & 0,01 & 0,02 & 2,62 & 0,01 & 0,01 \\
\hline $\mathrm{CV}$ & 158,98 & 31,65 & 216,69 & 147,90 & 47,66 & 211,69 & 137,81 & 63,39 & 184,56 \\
\hline Curtose & 7,40 & 3,13 & 13,11 & 5,90 & 0,66 & 6,06 & 7,34 & 1,43 & 2,59 \\
\hline Max & 11,00 & 0,03 & 0,24 & 11,00 & 0,03 & 0,10 & 11,00 & 0,04 & 0,05 \\
\hline Min & 0,20 & 0,01 & 0,00 & 0,20 & 0,01 & 0,00 & 0,20 & 0,01 & 0,00 \\
\hline
\end{tabular}

superficial $(0-20 \mathrm{~cm})$ da coleta realizada em dezembro de 2006. Observa-se, com isso que a precipitação pluvial da região tem sido suficiente para baixar os teores de $\mathrm{Cl}^{-}$no solo na estação chuvosa subsequente em virtude do lixiviamento desse elemento no solo.

\section{Conclusões}

Os valores de $\mathrm{pH}$ dos solos variaram ao longo do ano em estudo, ficando na faixa entre a neutralidade e a alcalinidade.

Ocorre um aumento em profundidade do teor de $\mathrm{Na}^{+}$trocável e da PST nos solos do Perímetro. 
As maiores concentrações de cátions e ânions solúveis estudados foram registradas nas camadas superiores.

Aproximadamente $12 \%, 14 \%$ e $28 \%$ dos lotes estudados estão afetados por sais nas profundidades de 0-20, 20-40 e $40-60 \mathrm{~cm}$, respectivamente.

\section{Referências}

ANDRADE, E. M. et al. Modelagem da concentração de íons no extrato de saturação do solo, na região da Chapada do Apodi. Revista Ciência Agronômica, Fortaleza, v. 33, n. 2, p. 25-32, 2002.

ANDRADE, E. M. et al. Evolução da concentração iônica da solução do solo em áreas irrigadas na Chapada do Apodi, CE. Revista Ciência Agronômica, Fortaleza, v. 35, n. 1, p. 9-16, 2004.

ANDRADE, T. S. et al. Variabilidade espaço-temporal da condutividade elétrica da água subterrânea na região semiárida de Pernambuco. Revista Brasileira de Engenharia Agrícola e Ambiental, Campina Grande, v. 16, n. 5, p. 496-504, 2012.

AYERS, R. S.; WESTCOT, D. W. A qualidade da água na agricultura. Tradução Hans R. Gheyi, José F. de Medeiros e Francisco A. Damasceno. 2. ed. Campina Grande: UFPB, 1999. 218 p. (Estudos FAO: Irrigação e Drenagem, n. 29).

BARRETO, A. N. et al. Condutividade hidráulica saturada em um solo aluvial do perímetro irrigado de São Gonçalo, PB. Revista Brasileira de Engenharia Agrícola e Ambiental, Campina Grande, v. 5, n. 1, p. 152-155, 2001

BATISTA, M. J. et al. Drenagem como instrumento de dessalinização e prevenção da salinização de solos. Brasília: Secretaria dos Recursos Hídricos, Ministério do Meio Ambiente, 2001. 203 p. (Série Informes Técnicos).

BEN-HUR, M. et al. Water and salt distribution in a field irrigated with marginal water under high water table conditions. Soil Science Society of America Journal, Madison, v. 65, n. 1, p. 65-191, 2001.

CAVAlCANTI, F. J. A. et al. Avaliação da fertilidade dos solos de Pernambuco: resultados de 1998. In: CONGRESSO BRASILEIRO DE CIÊNCIA DE SOLO, 27., 1999, Brasília. Anais... Brasília: Sociedade Brasileira de Ciência do Solo, 1999. CD-ROM.

CEUPPENS, J.; WOPEREIS, M. C. S. Impacto f non-drained irrigated rice cropping on soil salinization in the Senegal River Delta. Geoderma, Amsterdam, v. 92, n. 1-2, p. 125-140, 1999. http:// dx.doi.org/10.1016/S0016-7061(99)00034-8

CHAVES. L. H. G. et al. Variabilidade de propriedades químicas do solo aluvial da Ilha de Picos, Pernambuco. Revista de Biologia e Ciências da Terra, Paraíba, v. 6, n. 1, p. 13-19, 2006.

CHAVES, L. H. G. Propriedades químicas do solo aluvial da ilha de assunção - Cabrobó (Pernambuco). Revista Brasileira de Ciência do Solo, Viçosa, v. 28, n. 3, p. 431-437, 2004.

COELHO, M. A.; FERREYRA, F. F. Characterization of saltaffected soil in the semiarid region of Northeastern Brazil. Turrialba, San Jose, v. 36, n. 2, p. 171-178, 1986.

CORWIN, D. L. et al. Monitoring management-induced spatiotemporal changes in soil quality through soil sampling directed by apparent electrical conductivity. Geoderma, Amsterdam, v. 131, n. $3-4$, p. $369-387$, 2006. http://dx.doi.org/10.1016/j. geoderma.2005.03.014

CRUZ, M. G. M. Avaliação da qualidade das águas superficiais e subterrâneas do Distrito de Irrigação Jaguaribe-Apodi. 2001. 66 f. Dissertação (Mestrado em Engenharia Agrícola)-Universidade Federal do Ceará, Fortaleza, 2001.
DEPARTAMENTO DE OBRAS CONTRA AS SECAS - DNOCS. Estudos para a avaliação da disponibilidade hídrica do Açude Público Cachoeira II - Município de Serra Talhada - PE. Fortaleza, 1999. (Relatório apresentado ao DNOCS em julho de 1999).

EMATER. Sugestões de adubação para o Estado da Paraíba: $1^{\circ}$ aproximação. João Pessoa, 1979.

EMPRESA B R A ILEIRA DE PESQUIS A AGROPECUÁRIA - EMBRAPA. Manual de métodos de análise de solo. Rio de Janeiro: Centro Nacional de Pesquisa de Solos, 1997. $212 \mathrm{p}$.

EMPRESA B R A S I EIR A DE PES Q UIS A AGROPECUÁRIA - EMBRAPA. Serviço nacional de levantamento e conservação de solos: avaliação da fertilidade dos solos do Distrito Federal. Rio de Janeiro, 1980. 11 p. (Boletim Técnico, n. 74).

FREIRE, M. B. G. S.; FREIRE, F. J. Fertilidade do solo e seu manejo em solos afetados por sais. In: NOVAIS, R. F. et al. (Ed.). Fertilidade do solo. Viçosa: SBCS, 2007.

FREIRE, M. B. G. S. et al. Condutividade hidráulica de solos de Pernambuco em resposta à condutividade elétrica e RAS da água de irrigação. Revista Brasileira de Engenharia Agrícola e Ambiental, Campina Grande, v. 7, n. 1, p. 45-52, 2003a.

FREIRE, M. B. G. S. et al. Estimativa do risco de sodificação de solos de Pernambuco pelo uso de águas salinas. Revista Brasileira de Engenharia Agrícola e Ambiental, Campina Grande, v. 7, n. 2, p. 227-232, 2003b.

GHASSEMI, F.; JAKEMAN, A. J.; NIX, H. A. Salinization of land and water resources: human causes, extent, management and case studies. Wallingford: CAB International, 1995. $526 \mathrm{p}$.

GONÇALVES, I. V. C. et al. Alterações químicas de um Neossolo Flúvico irrigado com águas salinas. Revista Ciência Agronômica, v. 42, n. 3, p. 589-596, 2011.

HERRERO, J.; PÉREZ-COVETA o. Soil salinity changes over 24 years in a Mediterranean irrigated district. Geoderma, Amsterdam, v. 125 , n. 3-4, p. 287-308, 2005. http://dx.doi.org/10.1016/j. geoderma.2004.09.004

KHAN, M. A.; ABDULLAH, Z. Salinity-sodicity induced changes in reproductive physiology of rice (Oryza sativa L.) under dense soil conditions. Environmental and Experimental Botany, Paris, v. 49, n. 2, p. 145-157, 2003. http://dx.doi.org/10.1016/ S0098-8472(02)00066-7

MEDEIROS, J. F.; GHEYI, H. R. Manejo do sistema solo-águaplanta em solos afetados por sais. In: GHEYI, H. R.; QUEIROZ, J. E.; MEDEIROS, J. M. (Ed.). Manejo e controle da salinidade na agricultura irrigada. Campina Grande: UFPB: SBEA. p. 239-288 1997.

OLIVEIRA, J. J. et al. Variabilidade espacial de propriedades químicas em um solo salino-sódico. Revista Brasileira de Ciência do Solo, Viçosa, v. 23, n. 4, p. 783-789, 1999.

OLIVEIRA, J. J. Variabilidade de características químicas de um solo salino-sódico. 1998. 101 f. Dissertação (Mestrado)-Centro de Ciências e Tecnologia, Universidade Federal da Paraíba, Campina Grande, 1998.

PEREIRA, O. J.; MATIAS FILHO, J.; ANDRADE, E. M. Variação do teor de sais no solo irrigado por aspersão e ação da chuva na sua lixiviação. Revista Ciência Agronômica, Fortaleza, v. 17, n. 1, p. 61-65, 1986. 
PESSOA, L. G. M. Crescimento de cebola irrigada com águas salinas em solos do semiárido de Pernambuco. Revista Brasileira de Ciências Agrárias, Recife, v. 7, n. 3, p. 527-534, 2012. http:// dx.doi.org/10.5039/agraria.v7i3a1552

PÉREZ-SIRVENT, C. et al. The role of low-quality irrigation water in the desertification of semi-arid zones in Murcia, SE Spain. Geoderma, Amsterdam, v. 113, n. 1-2, p. 109-125, 2003. http:// dx.doi.org/10.1016/S0016-7061(02)00334-8

PIZARRO, F. Drenaje agrícola y recuperacion de suelos salinos. Madrid: Agrícola Española, 1978. 528 p.

RHOADES, J. D. Drainage for salinity control. In: VAN SCHILFGAARDE, J. (Ed.). Drainage for Agriculture. Madison: American Society of Agronomy, 1974. 700 p.

RICHARDS, L. A. Diagnosis and improvement of saline and alkali soils. Washington: United States Salinity Laboratory, 1954. 160 p. (United States Department of Agriculture Handbook, n. 60).

SALVIANO, A. A. C.; VIEIRA, S. R.; SPAROVEK, G. Variabilidade espacial de atributos de solo e de Crotalaria juncea L. em área severamente erodida. Revista Brasileira de Ciência do Solo, Viçosa, v. 22, n. 1, p. 115-122, 1998.

SANTOS, H.L. \& VASCONCELLOS, C.A. Determinação do número de amostras de solo para análise química em diferentes condições de manejo. Revista Brasileira de Ciência do Solo, v. 11. p. 97-100, 1987.

SILVEIRA, J. A. G. et al. Salt-induced effects on the nitrogen assimilation related to growth in cowpea plants. Environmental and Experimental Botany, Memphis, v. 46, n. 2, p. 171-179, 2001. http://dx.doi.org/10.1016/S0098-8472(01)00095-8

SILVA, P. C. M. Avaliação e variabilidade espacial de propriedades químicas do solo da "Extensão Maria Tereza" - Perímetro Irrigado Senador Nilo Coelho, Petrolina-PE. 2001. 104 f. Dissertação (Mestrado)-Universidade Federal da Paraíba, Campina Grande, 2001.

SOUSA, A. R. Solos da planície aluvial do Vale do Pajeú em Serra Talhada (PE): ambiente agrícola e uso da terra. 1995. 145 f. Tese (Doutorado em Solos e Nutrição de Plantas)-Universidade Federal de Viçosa, Viçosa, 1995.

SOUZA, L. C.; QUEIROZ, J. E.; GHEYI, H. R. Variabilidade espacial da salinidade de um solo aluvial no semi-árido paraibano. Revista Brasileira de Engenharia Agrícola e Ambiental, Campina Grande, v. 4, n. 1, p. 35-40, 2000.

SOUZA, L. S.; COGO, N. P.; VIEIRA, S. R. Variabilidade das propriedades físicas e químicas do solo em pomar de citros. Revista Brasileira de Ciência do Solo, Viçosa, v. 21, n. 3, p. 367-372, 1997.

VAN RAIJ, B. Fertilidade do solo e adubação. Piracicaba: Ceres: Potafos, 1991. 343 p.

WARRICK, A. W.; NIELSEN, D. R. Spatial variability of soil physical properties in the field. In: HILLEL, D. (Ed.). Applications of soil physics. New York, Academic Press, 1980. p. 319-344. http:// dx.doi.org/10.1016/B978-0-12-348580-9.50018-3 